\title{
Papillary neoplasm of the endolymphatic sac in a patient with von Hippel-Lindau disease
}

\author{
M B Delisle, E Uro, I Rouquette, E Yardeni, J L Rumeau
}

\begin{abstract}
Glandular tumours involving the middle ear and the mastoid are rare, and distinguishing between adenoma and adenocarcinoma is difficult. Two distinct histopathological patterns are accepted. While their clinical presentation differs, both require primary surgical treatment and both have a high rate of local recurrence. The papillary form takes a more aggressive course and wider regional spread. This pattern occurs predominantly in women. Its microscopic appearance and clinical course have been extensively described by Heffiner who considered it as "low-grade adenocarcinoma of probable endolymphatic sac origin". A few cases have been associated with von Hippel-Lindau disease. The case of a 32 year old black woman is described. It is suggested that papillary adenocarcinoma of the endolymphatic sac should be considered in the spectrum of neoplasms seen in von Hippel-Lindau disease.
\end{abstract}

(F Clin Pathol 1994;47:959-961)

Glandular tumours of the temporal bone are rare and the distinction between adenoma and adenocarcinoma is difficult. In 1990 Benecke et $a l^{1}$ differentiated two distinct histopathological patterns-mixed and papillary, with different clinical presentations, both with a high rate of local recurrence, requiring primary surgical treatment. The papillary pattern has a more aggressive course and wider regional impact (extension to facial nerve, bone and the dura mater, and invasion of the cerebellar posterior fossa). This pattern occurs predominantly in women. The microscopic appearance and clinical course have been outlined by Heffner, ${ }^{2}$ as "low-grade adenocarcinoma of probable endolymphatic sac origin". We had the opportunity of observing this type of tumour in a young woman with von Hippel-Lindau (VHL) disease. The rarity of these two diseases and the occurrence of such an association which has been reported in four similar cases ${ }^{134}$ emphasise the necessity of including this tumour in the spectrum of VHL disease.

\section{Case report}

A 29 year old black woman from Martinique was initially admitted for headaches and vertigo of recent onset. Radiographic pictures showed two tumours in the posterior cerebral fossa and an intradural mass at cervical level C3. The patient was referred to the neurosurgical department, where she underwent three successive surgical procedures: (1) resection of a haemangioblastoma of the left cerebellopontine angle; (2) resection of a partially cystic mass of the right cerebello-pontine angle, which had invaded the temporal bone into the tympanic cavity and the internal auditory canal; and (3) resection of an intramedullary cervical haemangioblastoma. Later, pancreatic cysts were discovered and VHL disease was diagnosed.

Two years later a computed tomogram and magnetic resonance imaging scan showed recurrence of the tumour of the right cerebello-pontine angle. A few weeks later the patient presented with right otorrhagia and otalgia. She was otherwise well and also pregnant. Pregnancy and delivery were normal. A haemorrhagic tumour, invading the mastoid antrum and encasing the third portion of the facial nerve, and extending to the jugular foramen, was partially resected. The postoperative course was uneventful. Further investigation of VHL disease showed an exclusively cystic lesion $1 \mathrm{~cm}$ in diameter on the anterior aspect of the right kidney and a small nodular area of hypofixation on the right lobe of the thyroid. The patient remained well five months after partial resection of the recurrent tumour of the right cerebello-pontine angle.

\section{Methods}

Tissue specimens for histological examination were fixed in $10 \%$ buffered formalin. Sections were stained with haematoxylin and eosin, periodic acid Schiff (PAS), and Perls's Prussian blue. Immunohistochemical studies were performed on paraffin wax embedded tissue using an avidin biotin peroxidase conjugate with monoclonal antibodies to epithelial membrane antigen (EMA) (1 in 50, Dako Corporation), vimentin ( 1 in 25, Dako), cytokeratin (MNF116, 1 in 100, Dako), glial fibrillary acidic protein (GFAP) ( 1 in 100 , Dako), and BNH9 ( 1 in $10, Y$ antigens to endothelial cells, Immunotech SA), and polyclonal antibodies to $S-100$ protein ( 1 in 400 , Dako), chromogranin ( 1 in 400 , Dako), and thyroglobulin ( 1 in 3000 , Dako). The reaction was developed using 3,3 diaminobenzidine.

\section{Pathological observations}

Microscopic examination of the left cerebellopontine angle and medullary tumours 


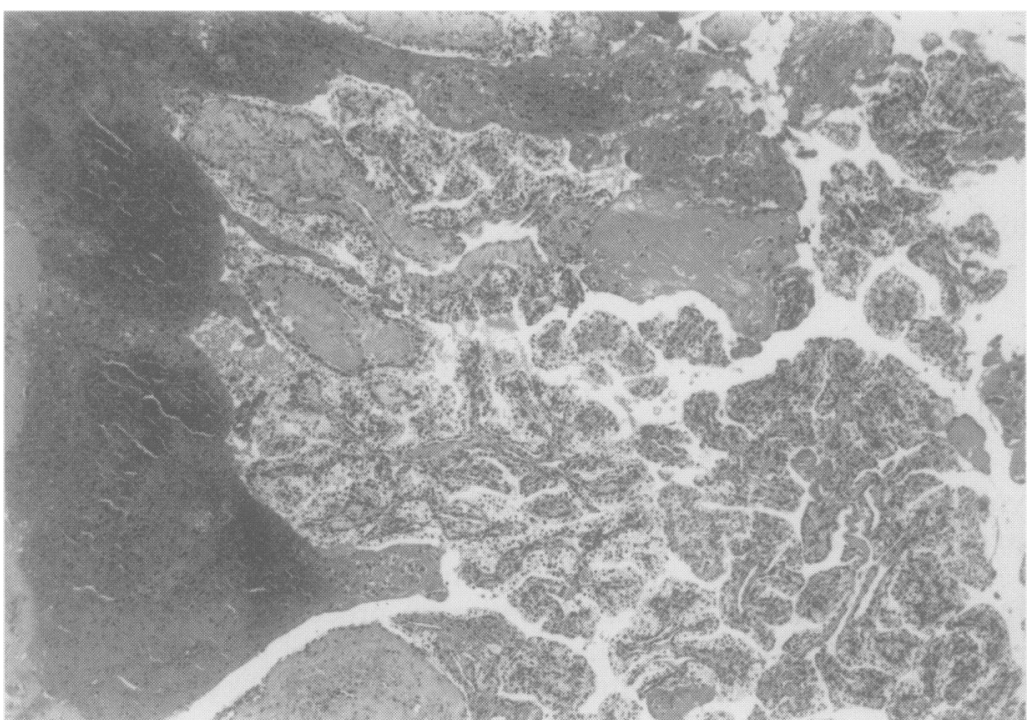

Figure 1 Right cerebello-pontine angle tumour (RCPAT): thin axes or thick hyalinised axes are found in papillae. Some cavities contain haemorrhagic material.

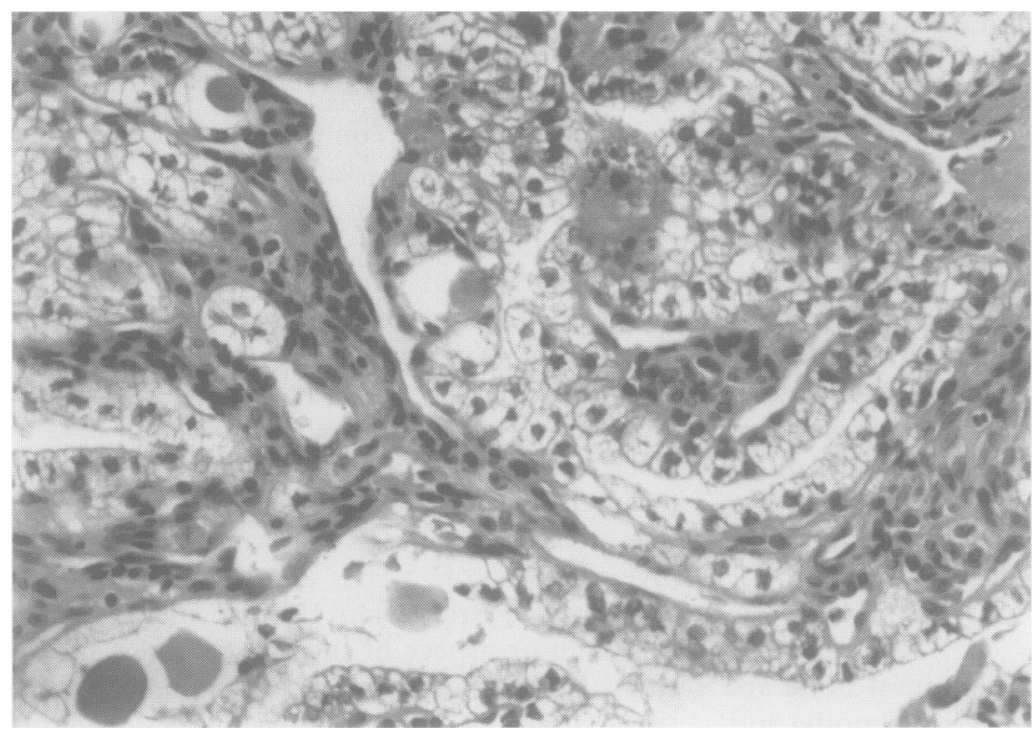

Figure 2 RCPAT: some colloid-like material is seen in the solid areas close to papillae with microvacuolated cells. cytoplasmic staining for EMA, cytokeratin, BNH 9, and S-100. A few cylindrical cells expressed vimentin, essentially at the basal level. Cytokeratin positivity was focal. No positive reaction was seen with GFAP, thyroglobulin, or chromogranin antibodies.

Reprocessing of the paraffin wax blocks from the initial tumour made it possible to find a few epithelial papillary structures with the same immunohistochemical phenotype, indicating that the initial lesion was already a papillary adenocarcinoma.

\section{Discussion}

Possible diagnoses for this tumour invading the middle ear, mastoid, and cerebral posterior fossa were haemangioblastoma, metastatic carcinoma of thyroid or renal origin, and primary adenomatous tumour. Clinically, haemangioblastoma, the most common lesion in VHL disease, had been suspected initially. The present tumour, highly vascularised and characterised by clear cells, did not present with the typical pattern of haemangioblastoma nor its immunocytochemical features. Metastatic tumour was also ruled out. Papillary as well as pseudofollicular structures containing proteinaceous material were reminiscent of thyroid carcinoma. Neither the typical nucleus of papillary thyroid carcinoma nor positivity for thyroglobulin antibody were found. Metastasis from renal carcinoma could also be excluded.

Primary glandular tumour remained the only possibility. Various terms have been used to describe this type of tumour when it affects the temporal bone. Apart from carcinoid tumour, also described as mixed or amphicrine tumours, because they contain cells with secretory activity and have solid trabecular or tubular glandular growth patterns, pure adenomatous benign and malignant neoplasms, have also been described. ${ }^{16-9}$ Among these, papillary or papillary cystic tumours, which are very rare, have been isolated and defined by their typical clinical, evolutive, and pathological features. ${ }^{127}$ They frequently occur in women and their initial clinical manifestations are hearing loss and vertigo of rather long duration. They extend to the petrous apex and frequently involve the middle or posterior cranial fossae, or both, with occasional extension to the facial nerve. They are papillary cystic tumours with single-layered cells covering the papillae. These cells are columnar or cuboidal with an optically clear cytoplasm and a nucleus pushed toward the apical end. Some tubular structures with small cysts filled with PAS stained material can be seen. Mitotic activity is rare. Hypocellular fibrotic areas with old and recent haemorrhage and cholesterol granuloma are often noted. These features were found in our case. Extension to the petrous apex and involvement of the posterior cranial fossa were noted from the initial lesion. Local recurrence within a few years with extension to the facial nerve was observed. The pathological data were quite characteristic. Vacuolated cells and papillary expressed in the "pseudo-colloidal" material. Some epithelial cells were positive for Perls's Prussian blue.

Immunohistochemistry showed diffuse 
structures were predominant: they are usually absent in middle ear adenomas. In these cells the nuclei were midcellular and apical, as reported in papillary adenocarcinomas. ${ }^{127}$ Recent and old haemorrhages were seen associated with areas of fibrosis and reactive changes. Iron positive granules in tumour cells and erythrocytes within gland lumina were conspicuous. This is a common feature of papillary carcinomas and ceruminous gland tumours which rarely invade the temporal bone. $^{6}$ Immunohistochemical results were consistent with those of the few previous reported studies. Tumour cells are positive with keratins, frequently positive with $S-100$, and less often with neuronal markers. ${ }^{257}$ EMA and vimentin have also been found to be positive, with vimentin staining restricted to the basal portion of cells. ${ }^{7}$

Papillary adenocarcinomas of temporal bone are characterised by local invasion with bone destruction, facial nerve involvement, and extension both to the posterior cerebellar fossa and the internal auditory canal. This regional aggressiveness is related to the histogenesis of the lesion, the origin of which has been debated. However, the derivation from endolymphatic sac epithelium proposed by Heffner $^{2}$ seems to be accepted now. The endolymphatic sac originates from the neuroectoderm (otocyst). It is situated between the dura and the posterior surface of the petrous portion of the temporal bone. Its epithelium is arranged in villous folds and lies on loose connective tissue containing blood vessels. These topographical and microscopic characteristics are strong arguments for an endolymphatic sac origin for this tumour as opposed to one of the middle ear. This further observation leads us to insist on the differenti- ation of this tumour from adenomas or mixed tumours arising in the middle ear cleft. The other point of interest is the association with VHL disease. A few such cases have been reported..$^{34510}$ In three of them the lesion was bilateral. ${ }^{35}$ This argues for recommending the inclusion of papillary carcinoma of the endolymphatic sac in the spectrum of neoplasms seen in VHL disease. Moreover, this otological manifestation can be the initial sign of the disease. ${ }^{3}$

We thank Dr A Jouvet (Pathology Department, Neurological Hospital, Lyon) for interesting suggestions, Professors Y Lazorthes (Neurosurgery Department, Rangueil University Hospital, Toulouse), B Fraysse (Otorhinolaryngology Department, Purpan University Hospital, Toulouse), and Dr B Gorguet (Pathology Department, Purpan University B Gorguet (Pathology Department, Purpan University
Hospital, Toulouse) for contributing clinical data and specimens.

1 Benecke JE, Noel FL, Carberry JN, House JW, Patterson $\mathrm{M}$. Adenomatous tumors of the middle ear and mastoid. Am F Otol 1990;11:20-6.

2 Heffner DK. Low-grade adenocarcinoma of probable endolymphatic sac origin: a clinicopathologic study of 20 cases. Cancer 1989;64:2292-302.

3 Eby TL, Makek MS, Fisch U. Adenomas of the temporal bone. Ann Otol Rhinol Laryngol 1988;97:605-12.

4 Palmer JM, Coker NJ, Harper RL. Papillary adenoma of the temporal bone in Von Hippel-Lindau disease. Otolaryngol Head Neck Surg 1989;100:64-8.

5 Poe DS, Tarlov EC, Thomas CB, Kveton JF. Aggressive papillary tumors of temporal bone. Otolaryngol Head Neck Surg 1993;108:80-6.

6 Faverly DRGS, Manni JJ, Smedts F, Verhofstad AAJ, Van Haelst UJGM. Adenocarcinoid or amphicrine tumors of the middle ear a new entity? Path Res Pract 1992; 188: $162-71$.

7 Gaffey MJ, Mills SE, Fechner RE, Intemann SR, Wick MR. Aggressive papillary middle-ear tumor: a clinicoMR. Aggressive papillary middle-ear tumor: a clinicopathologic entity distinct from

8 Mills SE, Fechner RE. Middle ear adenoma: a cytologically uniform neoplasm displaying a variety of architectural patterns. Am $\mathcal{F}$ Surg Pathol 1984;8:677-85.

9 Pallanch JF, McDonald TJ, Weiland LH, Facer GW, Harner SG. Adenocarcinoma and adenoma of the middle ear. Laryngoscope 1982;92:47-54.

10 Batsakis JG. Papillary neoplasms (Heffner's tumors) of the endolymphatic sac. Ann Otol Rhinol Laryngol 1993;102: 646-51.

\section{University \\ Department of \\ Pathology, The \\ Medical School, Beech \\ Hill Road, Sheffield, \\ S10 2RX \\ J A Lee \\ Department of ENT, \\ Royal Hallamshire \\ Hospital, Sheffield \\ A Panarese \\ Correspondence to: \\ Dr Lee \\ Accepted for publication \\ 19 April 1994}

\begin{abstract}
Endochondral pseudocyst of the auricle is an uncommon, though distinctive clinicopathological entity occurring mainly in young men. An additional case is reported and the differential diagnosis and pathogenesis discussed. It is suggested that lymphatic dilatation of normally present tissue planes could be the most likely causative mechanism. Minor trauma to susceptible ears also seems to be a requirement for development of this condition.
\end{abstract}

(F Clin Pathol 1994;47:961-963)
Endochondral pseudocyst of the auricle is an uncommon condition which presents as a painless, dome-shaped, cystic swelling on the anterior surface of the upper part of the auricle. ${ }^{1}$ Although originally reported in Chinese people, ${ }^{2}$ it can occur in any race. ${ }^{1}$ All ages may be affected, but the lesion occurs primarily in young adults and shows a strong male predominance ( $90 \%$ of cases). Ten to 20 per cent of patients develop a similar lesion on the contralateral pinna, usually asynchronously. A needle aspirate produces $0.5-10 \mathrm{ml}$ of straw coloured, viscous, albumin containing fluid with osmolarity, glucose, and protein concentrations similar to those of serum. ${ }^{1-3}$ 